\title{
Is dexmedetomidine the gold standard for pediatric procedural sedation and anxiolysis?
}

\author{
Eduardo Mekitarian Filho ${ }^{1 *}$ \\ ${ }^{1} \mathrm{MD}, \mathrm{MSc}$, PhD, Pediatric Intensive Care Unit, Universidade de São Paulo, São Paulo, SP, Brazil \\ Study conducted at Pediatric Intensive Care Unit, Universidade de São Paulo, São Paulo, SP, Brazil \\ Article received: $7 / 28 / 2016$ \\ Accepted for publication: 10/19/2016 \\ *Correspondence: \\ Unidade de Terapia Intensiva Pediátrica, USP \\ Address: Av. Dr. Enéas de Carvalho Aguiar, 647 \\ São Paulo, SP - Brazil \\ Postal code: 05467-000 \\ emf2002@uol.com.br
}

Pediatric procedural sedation is a growing issue in the emergency setting, and finding the right drug to perform safe and effective sedation is still a challenge. I would like to discuss the article "Double-blind randomized controlled trial of intranasal dexmedetomidine versus intranasal midazolam as anxiolysis prior to pediatric laceration repair in the emergency department," by Neville et al., which is currently in press in the Academic Emergency Medicine Journal. The authors randomized 38 children to receive either intranasal dexmedetomidine (DEX) or intranasal midazolam before laceration repairs, and chose as primary outcome the anxiety score at the time of patient positioning for the repair. The proportion of patients who were classified as not anxious at the position for procedure was significantly higher in the dexmedetomidine group (70\%) versus the midazolam group (11\%). Authors concluded that intranasal DEX is an alternative with good results for anxiolysis prior to painful procedures in children compared to midazolam.

DEX is a highly selective $\alpha 2$ adrenergic agonist that offers some unique and unmatched sedation characteristics. ${ }^{2}$ Without pediatric labeling, DEX has been studied for pediatric sedation and anxiolysis, intravenously or using other administration routes, such as intranasal (IN). In contrast to all other sedatives, DEX produces a sleep somnolence state which closely resembles that of non-REM sleep on electroencephalogram. ${ }^{3}$ DEX maintains spontaneous ventilation, has minimal respiratory effects and preserves upper airway tone, making it an attractive choice for pediatric procedural sedation and anxiolysis.

The majority of pediatric sedation literature on DEX described its application for non-painful radiological imaging studies such as MRI, computerized tomography scans, and nuclear medicine studies. A few studies addressed this sedative for anxiolytic purposes. Some authors studied DEX alone or carried out clinical trials comparing it with other drugs. Recently, Sidhu et al. ${ }^{4}$ studied 105 ASA 1-2 surgical patients comparing IN DEX with IN clonidine. Using an initial dose of $2 \mathrm{mcg} / \mathrm{kg}$ of IN DEX, satisfactory anxiolysis was achieved in $88.5 \%$ of these patients and in $60 \%$ of the clonidine patients, with significantly less rescue analgesia requirements in the DEX group. Another recent and very interesting study was conducted by Yao et al..$^{5}$ with 90 children receiving $1-2 \mathrm{mcg} / \mathrm{kg}$ of IN DEX prior to laryngeal mask insertion, which concluded that patients receiving $2 \mathrm{mcg} / \mathrm{kg}$ had significant lower alveolar concentrations of sevoflurane prior to the procedure and less emergency delirium after it.

The two studies above described were the only ones focused on DEX premedication in children, prior to two meta-analyses ${ }^{6,7}$ published in 2014 that verified the efficacy and safety of premedication with DEX in children, alone or associated with midazolam. Together, the authors pooled 24 randomized controlled trials and concluded that DEX is superior to midazolam premedication because it resulted in enhanced preoperative sedation and decreased postoperative pain. In addition, DEX premedication provided clinical benefits that included reduced requirements for rescue analgesia and reduced agitation or delirium and shivering during the postoperative period.

Our group has previously studied IN DEX and midazolam for pediatric procedural sedation, and we felt that the quality of anxiolysis and sedation provided by IN DEX ${ }^{8,9}$ was far superior. However, we selected two prospective cohorts and our primary outcomes were time to sedation and rates of failed sedation. As we didn't 
randomize the patients, comparison between the drugs is flawed, but no failed sedations occurred in the DEX patients, and no adverse events with clinical relevance were observed. Parent satisfaction, although not directly measured by Neville et al., ${ }^{1}$ was also greater with IN DEX.

One concern with the study conducted by Neville et al. ${ }^{1}$ was that the anxiety score at positioning for procedure of the patients receiving DEX was 9.2 points lower than that of the patients receiving midazolam, according to the modified Yale Preoperative Anxiety Scale, which the authors used as a reference. Although other baseline characteristics between the two groups (DEX and midazolam) were similar, these random findings can cause a potential bias to the final conclusions. Despite probably being a better sedative than midazolam, one could conclude that DEX performed better in these patients because of their baseline anxiety conditions.

I believe that larger studies with IN DEX as premedication are needed in order to find the better option for pediatric anxiolysis. The article from Neville et al. substantiates the indication of IN DEX as a sedative of choice, with minimal adverse events and good parent and staff satisfaction.

\section{Conflict of interest}

The author declares no conflict of interest.

\section{References}

1. Neville DN, Hayes KR, Ivan Y, McDowell ER, Pitetti RD. Double-blind randomized controlled trial of intranasal dexmedetomidine versus intranasal midazolam as anxiolysis prior to pediatric laceration repair in the emergency department. Acad Emerg Med. 2016; 23(8):910-7.

2. Mahmoud MA, Mason KP. A forecast of relevant pediatric sedation trends. Curr Opin Anesthesiol. 2016; 29(Suppl 1):S56-67.

3. Mason KP, Lerman J. Dexmedetomidine in children: current knowledge and future applications. Anesth Analg. 2011; 113(5):1129-42.

4. Sidhu GK, Jindal S, Kaur G, Singh G, Gupta KK, Aggarwal S. Comparison of intranasal dexmedetomidine with intranasal clonidine as a premedication in surgery. Indian J Pediatr. 2016; 83(11):1253-8.

5. Yao Y, Qian B, Lin Y, Wu W, Ye H, Chen Y. Intranasal dexmedetomidine premedication reduces minimum alveolar concentration of sevoflurane for laryngeal mask airway insertion and emergence delirium in children: a prospective, randomized, double-blind, placebo-controlled trial. Paediatr Anaesth. 2015; 25(5):492-8.

6. Peng K, Wu SR, Ji FH, Li J. Premedication with dexmedetomidine in pediatric patients: a systematic review and meta-analysis. Clinics (Sao Paulo). 2014; 69(11):777-86.

7. Sun Y, Lu Y, Huang Y, Jiang H. Is dexmedetomidine superior to midazolam as a premedication in children? A meta-analysis of randomized controlled trials. Paediatr Anaesth. 2014; 24(8):863-74.

8. Mekitarian Filho E, Robinson F, de Carvalho WB, Gilio AE, Mason KP. Intranasal dexmedetomidine for sedation for pediatric computed tomography imaging. J Pediatr. 2015; 166(5):1313-5.

9. Mekitarian Filho E, de Carvalho WB, Gilio AE, Robinson F, Mason KP Aerosolized intranasal midazolam for safe and effective sedation for quality computed tomography imaging in infants and children. J Pediatr. 2013; 163(4):1217-9. 